\title{
On the solution of Wave-Schrodinger equation
}

\section{Wanchak Satsanit}

Department of Mathematics, Faculty of Science, Maejo University, Chiang Mai 50290, Thailand.

\begin{abstract}
In this paper, we are finding a solution of the fractional Wave-Schrodinger equation by Laplace transform in the sense of Caputo fractional derivative. It was found that the fundamental solution of the equation is related to Wright function.
\end{abstract}

Keywords: Dirac delta distribution, Laplacian operator, Wright function.

2010 MSC: 46F10, 46F12.

(C)2020 All rights reserved.

\section{Introduction}

The Laplacian operator $\triangle^{k}$ iterated $k$-times is defined by

$$
\triangle^{k}=\left(\frac{\partial^{2}}{\partial x_{1}^{2}}+\frac{\partial^{2}}{\partial x_{2}^{2}}+\cdots+\frac{\partial^{2}}{\partial x_{n}^{2}}\right)^{k},
$$

where $n$ is the dimension of space $\mathbb{R}^{n}$ and $k$ is a non-negative integer. Kananthai [1] has proved that the generalized function $(-1)^{k} S_{2 k}(x)$ is an elementary solution of the operator $\Delta^{k}$, that is

$$
\triangle^{k}(-1)^{k} S_{2 k}(x)=\delta,
$$

where $\delta$ is the Dirac-delta distribution and $S_{2 k}(x)$ is defined by

$$
S_{2 k}(x)=\frac{\pi^{-\frac{n}{2}} 2^{-2 k} \Gamma\left(\frac{n-2 k}{2}\right)\left(x_{1}^{2}+x_{2}^{2}+\cdots+x_{n}^{2}\right)^{\frac{2 k-n}{2}}}{\Gamma(k)} .
$$

In 2002, Kananthai et al. [2] have first introduced the operator $\triangle_{i}^{k}$ and is defined by

$$
\triangle_{i}^{k}=\left(\sum_{i=1}^{p} \frac{\partial^{2}}{\partial x_{i}^{2}}+i \sum_{j=p+1}^{p+q} \frac{\partial^{2}}{\partial x_{j}^{2}}\right), i=\sqrt{-1} .
$$

Email address: wanchack@gmail.com (Wanchak Satsanit)

doi: $10.22436 /$ jnsa.013.04.01

Received: 2019-07-09 Revised: 2019-10-31 Accepted: 2019-11-13 
They have proved the function $(-1)^{\mathrm{k}}(-i)^{\frac{q}{2}} S_{2 k}(x)$ is an elementary solution of the operator $\triangle_{i}^{k}$ and $S_{2 k}(x)$ is defined by (1.1). It is well known that the linear Schrodinger equation can be written as the following form

with the initial condition

$$
\frac{\partial}{\partial t} \mathfrak{u}(x, t)=i \frac{\partial^{2}}{\partial x^{2}} \mathfrak{u}(x, t), i=\sqrt{-1}
$$

$$
u(x, 0)=f(x) .
$$

The Schrodinger equation has been widely in application in science and engineering, there are several integral transforms such as Laplace transform, Fourier transform, Wavelet transform, etc., for solving the equation.

The purpose of this work is to introduce a new function where related the Wright function $[3,4]$ and studied Laplace transform of a new function. After that, we are solving the fundamental solution of the Wave-Schrodinger equation as follows:

$$
\frac{\partial^{\alpha}}{\partial t^{\alpha}} \phi(x, t)+i \frac{\partial^{2}}{\partial x^{2}} \phi(x, t)=0, i=\sqrt{-1}, 1<\alpha \leqslant 2,
$$

with the initial condition

$$
\phi(x, 0)=0, \phi_{\mathfrak{t}}(x, 0)=\delta(x),
$$

where $\delta$ is the Dirac-delta distribution and $\frac{\partial^{\alpha}}{\partial t^{\alpha}}$ is the Caputo derivative. Before going that point, the following definitions and some important concepts are needed.

\section{Preliminaries}

Definition 2.1. Let $f(t)$ be a function an exponential order and piecewise continuous. The Laplace transform of the function $f$ is given by

$$
\mathcal{L}[\mathbf{f}(\mathrm{t})]=\int_{0}^{\infty} e^{-s t} f(t) d t .
$$

Definition 2.2. Let $f(t)$ be a function of the Schwart space, the Fourier transform of $f(t)$ is given by

$$
\widehat{f}(w)=\int_{\mathbb{R}} f(t) e^{i w t} d t .
$$

Definition 2.3. For $m$ to be the smallest integer that exceeds $\alpha$, the Caputo fractional derivatives of order $\alpha$ is defined by

$$
D^{\alpha} u(x, t)=\frac{\partial^{\alpha} \mathfrak{u}(x, t)}{\partial t^{\alpha}}=\left\{\begin{array}{l}
\frac{1}{\Gamma_{(m-\alpha)}} \int_{0}^{t}(t-\tau)^{m-n-1} \frac{\partial^{m}}{\partial t^{m}} \mathfrak{u}(x, t) \\
\frac{\partial^{m}}{\partial t^{m}} \mathfrak{u}(x, t), n=m .
\end{array}\right.
$$

Definition 2.4. The Laplace transform of the Caputo fractional derivative is defined by

$$
\mathcal{L}\left[D^{\alpha} f(t)\right]=s^{\alpha} F(s)-\sum_{k=0}^{n-1} s^{\alpha-k-1} f^{(k)}(0), n-1<\alpha<n .
$$

Definition 2.5. The Wright function $W_{\alpha, \beta}$ is defined by

$$
W_{\alpha, \beta}=\sum_{n=0}^{\infty} \frac{Z^{n}}{n ! \Gamma(n \alpha+\beta)}, \alpha>-1, \beta \in \mathbb{C},
$$

where $\Gamma(x)$ is the Euler Gamma function given by the integral

$$
\Gamma(x)=\int_{0}^{\infty} t^{x-1} e^{-6} d t
$$


Lemma 2.6. The function $\gamma(\mathrm{a}, \mathrm{t})$ is defined by the following expressions

$$
\gamma(a, t)=t^{-\alpha+1} W_{-\alpha, 2-\alpha}\left(a t^{-\alpha}\right)
$$

and Laplace transform of $\gamma(\mathrm{a}, \mathrm{t})$ is given by

$$
\mathcal{L}[\gamma(a, t)]=s^{\alpha-2} e^{a s^{\alpha}} .
$$

Proof. By (2.1), we have

$$
\begin{aligned}
\mathcal{L}[\gamma(a, t)] & =\int_{0}^{\infty} e^{-s t} t^{-\alpha+1} W_{-\alpha, 2-\alpha}\left(a t^{-\alpha}\right) d t \\
& =\int_{0}^{\infty} e^{-s t} t^{-\alpha+1} \sum_{k=0}^{\infty} \frac{\left(a t^{-\alpha}\right)^{k}}{k ! \Gamma(-\alpha k+2-\alpha)} d t \\
& =\sum_{k=0}^{\infty} \frac{a^{k}}{k ! \Gamma(-\alpha k+2-\alpha)} \int_{0}^{\infty} e^{-s t} t^{-\alpha-\alpha k+1} d t \\
& =\sum_{k=0}^{\infty} \frac{a^{k}}{k ! \Gamma(-\alpha k+2-\alpha)} \mathcal{L}\left[t^{-\alpha-\alpha k+1}\right] \\
& =\sum_{k=0}^{\infty} \frac{a^{k}}{k ! \Gamma(-\alpha k+2-\alpha)} \frac{\Gamma(-\alpha-\alpha k+2)}{s^{-\alpha-\alpha k+2}} \\
& =\sum_{k=0}^{\infty} \frac{a^{k}}{k ! s^{-\alpha-\alpha k+2}}=s^{\alpha-2} \sum_{k=0}^{\infty} \frac{\left(a s^{\alpha}\right)^{k}}{k !}=s^{\alpha-2} e^{a s^{\alpha}} .
\end{aligned}
$$

That completes the proof.

\section{Main results}

Theorem 3.1. Consider the Fractional Wave-Schrodinger equation

$$
\frac{\partial^{\alpha}}{\partial t^{\alpha}} \phi(x, t)+i \frac{\partial^{2}}{\partial x^{2}} \phi(x, t)=0, i=\sqrt{-1}, 1<\alpha \leqslant 2
$$

with the initial condition

$$
\phi(x, 0)=0, \phi_{t}(x, 0)=\delta(x),
$$

where $\delta(x)$ is the dirac delta distribution. By the Laplace and Fourier transform we obtain that the fundamental solution of the equation (3.1) is given by

$$
\phi(x, t)=\frac{1}{2} \sqrt{i t}^{-\frac{\alpha}{2}+1} W_{-\frac{\alpha}{2}}, 2-\frac{\alpha}{2}\left(-\sqrt{i}|x| t^{-\frac{\alpha}{2}}\right),
$$

where $W_{\alpha, \beta}$ is the Wright function is defined by (2.2). If we put $\alpha=2$ in (3.1) the fractional Wave-Schrodinger equation reduces to

$$
\frac{\partial^{2}}{\partial t^{2}} \phi(x, t)+i \frac{\partial^{2}}{\partial x^{2}} \phi(x, t)=0
$$

and the solution of (3.2) is given by

$$
\phi(x, t)=\frac{1}{2} \sqrt{i} W_{-1,1}\left(-\sqrt{i}|x| t^{-1}\right) .
$$


Proof. By (3.1), we have

$$
\frac{\partial^{\alpha}}{\partial t^{\alpha}} \phi(x, t)+i \frac{\partial^{2}}{\partial x^{2}} \phi(x, t)=0
$$

Taking Laplace transform on both sides of (3.3) and and using definition 2.1 we get

$$
\begin{aligned}
\mathcal{L}\left[\frac{\partial^{\alpha}}{\partial t^{\alpha}} \phi(x, t)\right]+i \mathcal{L}\left[\frac{\partial^{2}}{\partial x^{2}} \phi(x, t)\right] & =0, \\
s^{\alpha} \phi(x, s)-s^{\alpha-2} \delta(x) & =-i \frac{\partial^{2}}{\partial x^{2}} \phi(x, s) .
\end{aligned}
$$

Applying Fourier transform respect to variable $x$ on both sides of (3.4), we obtain

$$
\begin{aligned}
s^{\alpha} \mathcal{F} \phi(x, s)-s^{\alpha-2} \mathcal{F}[\delta(x)] & =-i \mathcal{F} \frac{\partial^{2}}{\partial x^{2}} \phi(x, s), \\
s^{\alpha} \phi(\omega, s)-s^{\alpha-2} & =i \omega^{2} \phi(\omega, s), \\
\phi(\omega, s) & =\frac{s^{\alpha-2}}{s^{\alpha}+(-i) \omega^{2}}=\frac{i s^{\alpha-2}}{i s^{\alpha}+\omega^{2}} .
\end{aligned}
$$

Applying inverse Fourier transform on both sides of (3.5), we obtain

$$
\phi(x, s)=\mathcal{F}^{-1}\left[\frac{i s^{\alpha-2}}{i s^{\alpha}+\omega^{2}}\right]=\frac{\sqrt{i s^{\alpha-2}} e^{-|x| \sqrt{i s} \frac{\alpha}{2}}}{2 s^{\frac{\alpha}{2}}}=\frac{1}{2} \sqrt{i s^{\frac{\alpha}{2}-2}} e^{-|x| \sqrt{i s} \frac{\alpha}{2}} .
$$

By Lemma 2.6, we obtain the solution of (3.1) as follows

$$
\phi(x, t)=\frac{1}{2} \sqrt{i} r(-\sqrt{i}|x|, t)=\frac{1}{2} \sqrt{i} t^{-\frac{\alpha}{2}+1} W_{-\frac{\alpha}{2}, 2-\frac{\alpha}{2}}\left(-\sqrt{i}|x| t^{-\frac{\alpha}{2}}\right) .
$$

If we put $\alpha=2$ in (3.1) and (3.6), respectively, the equation reduces to the Wave-Schrodinger equation

$$
\frac{\partial^{2}}{\partial t^{2}} \phi(x, t)+i \frac{\partial^{2}}{\partial x^{2}} \phi(x, t)=0
$$

and the solution of (3.7) is given by

$$
\phi(x, t)=\frac{1}{2} \sqrt{i} W_{-1,1}\left(-\sqrt{i}|x| t^{-1}\right) .
$$

This completes the proof.

\section{Acknowledgment}

The authors would like to thank the Thailand Research Fund, Graduate School, Maejo University, Chiang Mai, Thailand, for financial support and Prof. Luis Guillermo Romero for the helpful1 discussion.

\section{References}

[1] A. Kananthai, On the Solution of the n-Dimensional Diamond Operator, Appl. Math. Comput., 88 (1997), 27-37. 1

[2] A. Kananthai, S. Suantai, V, Longani, On the operator $\oplus^{\mathrm{k}}$ related to the wave equation and Laplacian, Appl. Math. Comput., 132 (2002), 219-229. 1

[3] I. Podlubny, Fractional Differential Equations, Acedemic Press, San Diego, (1999). 1

[4] L. G. Romero, A generalization of the Laplacian operator, Palest. J. Math., 5 (2016), 204-207. 1 